\section{Süße Seiten}

Wenn Sie sich schon einmal gefragt haben, welche Strukturen sich hinter den in der Kohlenhydratchemie üblichen Sequenzangaben wie $\alpha$-D-Gal $p$ NAc$(1 \rightarrow 3)$-[ $\alpha$-L-Fuc $p-(1 \rightarrow 2)]-\mathrm{D}-\mathrm{Gal} p \quad$ verbergen, dann werden Sie auf der Homepage der von C.-W. von der Lieth geleiteten Molecular-Modeling-Gruppe des Deutschen Krebsforschungszentrums (DKFZ) fündig. Über eine schlichte Eingangsseite gelangt man zu einer Reihe von Service- und Informationsangeboten, die sich an Interessierte der Glycowissenschaften richten.

Das Programm SWEET-II ${ }^{[1]}$ dürfte dabei für den Besucher der Homepage die interessanteste Anwendung sein. SWEET-II erzeugt aus der üblichen Zuckernomenklatur 3D-Strukturen der eingegebenen Oligosaccharide in einer möglichen Konformation. Die Eingabe der Sequenz eines Kohlenhydrats erfolgt über eine Maske, die - je nach Erfahrung des Nutzers - in verschiedenen Ausführungen zur Verfügung steht. So lassen sich einfache Disaccharide aber auch komplexe, verzweigte Oligosaccharide und Glycokonjugate eingeben, wie z. B. Glycopeptide und -lipide. Auch Cyclodextrine werden akzeptiert. Wer mit der Zuckernomenklatur weniger vertraut ist, kann auch vorgefertigte biologisch relevante Oligosaccharide aus einer reichhaltigen Auswahl (Sphingolipide, Lipopolysaccharide, GPI-Anker, CoreStrukturen von $\mathrm{N}$ - und $\mathrm{O}$-Glycoproteinen, antigene Determinanten) anklikken. SWEET-II verknüpft entsprechende Monosaccharid-Template und optimiert anschließend jede glycosidische Bindung. Hieran schließt sich eine Ver- feinerung unter Verwendung von Allingers MM3-Kraftfeld an. Die Ausgabe der erzeugten Strukturen erfolgt im PDB- oder VRML-Format. Zur Visualisierung stehen verschiedene Optionen bereit. Mit dem frei erhältlichen Plugin Chime beispielsweise können die Strukturen am Bildschirm beliebig gedreht, skaliert und von allen Seiten betrachtet werden (Abbildung 1). Die von SWEET-II erzeugten Strukturen werden von den Autoren als vorläufig, jedoch zuverlässig bezeichnet und eignen sich als sinnvoller Ausgangspunkt für weitere Verfeinerungen.

Mit SWEET-II erhaltene oder beliebige andere PDB-Dateien können aber auch als Eingabe für ein weiteres Programm (PDB2MultiGIF) ${ }^{[2]}$ verwendet werden, das aus den kartesischen Koordinaten animierte GIF-Bilder von sich drehenden Molekülen erzeugt. Bei der Wahl der Darstellungsparameter (z. B. Kalottenmodell oder Kugel-Stab-Modell) ist man ist man recht frei. Grundkenntnisse des Visualisierungsprogramms RASMOL sind hierbei hilfreich.

Eine weitere Anwendung von potenziell großem Nutzen für Forschende aus dem Bereich der Glycobiologie ist die Datenbank SWEET-DB. ${ }^{[3]}$ Mit dieser Datensammlung, die sich momentan noch in der Aufbauphase befindet, wird der Versuch unternommen, aus verschiedenen Quellen verfügbare Informationen über Glycane über eine einheitliche Beschreibung der chemischen Struktur (Linear Notation for Unique Description of Carbohydrate Sequences, LINUCS) miteinander zu verknüpfen. Derzeit sind etwa 50000 Oligosaccharidstrukturen aus CarbBank ${ }^{[4]}$ und $1600{ }^{1} \mathrm{H}$ und ${ }^{13} \mathrm{C}-\mathrm{NMR}$-Spektren aus SugaBase ${ }^{[5]}$ enthalten. Dazu wurden die räumlichen Koordinaten (mit SWEET-II generiert und mit MM3 optimiert) sowie Links zu PubMed und dem Literaturlieferservice

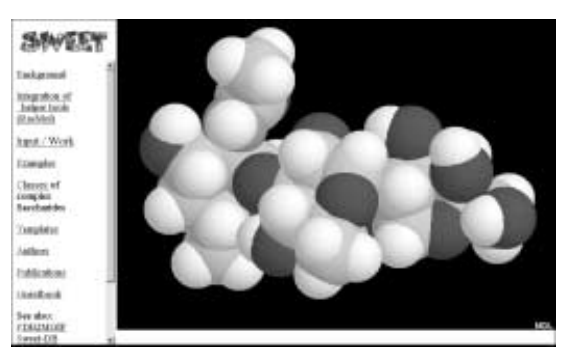

Abbildung 1. Ohne große Mühe: optimierte Struktur von $\alpha$-D-Gal $p$ NAc- $(1 \rightarrow 3)-[\alpha$-L-Fuc $p$ $(1 \rightarrow 2)]-\mathrm{D}-\mathrm{Gal} p$
Subito hinzugefügt. Über eine Suchmaske kann nach (Teil)strukturen und bibliographischen Daten recherchiert werden. Da im Moment die Programmoberfläche von SWEET-DB erneuert wird, war zum Zeitpunkt dieser Rezension die Suche nach NMR-Daten noch nicht wieder möglich. Leider ist der Datenbestand auch nicht ganz aktuell; die neuesten Publikationen stammen aus dem Jahr 1999, dem Jahr, in dem CarbBank eingestellt wurde. Derzeit wird allerdings an Methoden gearbeitet, die Datenbank zu aktualisieren, sodass in Zukunft mit einer äußerst interessanten Informationsquelle zu rechnen ist.

Abgerundet wird das Angebot der Website durch einige Hilfsprogramme, z. B. zur Konvertierung von Torsionswinkeln und zur Auswertung von Massenspektren von Glycoproteinen sowie eine Reihe nützlicher Links zu den Themen Kohlenhydrate, Molecular Modeling und Software.

An wen richtet sich diese Seite? Während die mit SWEET-II erzeugten Strukturen vermutlich eher in der Lehre zum Einsatz kommen dürften, könnte sich insbesondere SWEET-DB zu einem wichtigen Werkzeug für Forscher auf dem Gebiet der "Glycomics" entwickeln. Eine ähnliche Datenbank wird beispielsweise auch von dem Consortium for Functional Glycomics, ${ }^{[6]}$ an dem die Heidelberger Modeling-Gruppe teilnimmt, aufgebaut. Das eingangs erwähnte Trisaccharid ist übrigens die Erkennungsdeterminante der Blutgruppe A.

Valentin Wittmann Universität Frankfurt/M.

[1] A. Bohne, E. Lang, C.-W. von der Lieth, J. Mol. Model. 1998, 4, 33-43.

[2] A. Bohne, J. Mol. Model. 1998, 4, 344-346.

[3] A. Loß, P. Bunsmann, A. Bohne, A. Loß, E. Schwarzer, E. Lang, C.-W. von der Lieth, Nucleic Acids Res. 2002, 30, 405-408.

[4] S. Doubet, K. Bock, D. Smith, A. Darvill, P. Albersheim, Trends Biochem. Sci. 1989, 14, 475-477.

[5] J. van Kuik, K. Hard, J. F. Vliegenthart, Carbohydr. Res. 1992, 235, 53-68.

[6] http://glycomics.scripps.edu/ 